\title{
Christine Friederici
}

\section{Welect GmbH - Die andere Art von Werbung}

„Wir wollen, dass Menschen gerne Werbung sehen“ - dieses Ziel veranlasste Philipp Dommers und Olaf Peters-Kim im März 2016 dazu, das Adtech-Unternehmen Welect GmbH zu gründen. Der Wunsch, dass Nutzer mit Werbung etwas Positives verbinden, stand auch hinter der App WelectGo, welche bereits im November desselben Jahres gelauncht wurde. Die weltweit erste App für werbefinanzierte Tickets im öffentlichen Personennahverkehr wurde zu einem großen Erfolg und schaffte es im Dezember 2016 in die Top 10 der AppStore-Charts.

Gut zweieinhalb Jahre nach der Gründung liegt der Fokus des Unternehmens auf dem Internetdienst WelectPublish. Durch die Zusammenarbeit mit verschiedenen Verlagen wird den Nutzern ein selbstbestimmter Werbekonsum ermöglicht. So können Leser zum Beispiel die Arbeit der Redakteure bewusst honorieren oder einen Zugriff auf redaktionelle Online-Inhalte erhalten, die hinter den üblichen Barrieren wie Paywall, AdBlock-, Anmelde- und Aboschranken verborgen sind. Während die Nutzer selbst entscheiden können, ob und wenn ja, welche Werbung sie sehen wollen, bietet WelectPublish Verlagen eine neue Erlösquelle. Seit der Einführung des Produkts im Juli 2017 sind unter anderem Portale wie Rheinische Post Online, FOCUS Online und CHIP Partner geworden. Da sich die Nutzer aktiv dafür entscheiden, ein bestimmtes Werbevideo zu sehen und diesem die entsprechende Aufmerksamkeit schenken, profitieren als dritte Zielgruppe zudem die Werbetreibenden von WelectPublish.

Das Adtech-Start-up aus Düsseldorf konnte bereits in den vergangenen Jahren Verbraucher, Partner und Werbetreibende von seinen Produkten überzeugen. In der Anfangsphase des Unternehmens wurde vor allem der regionale Markt bedient. In den vergangenen Jahren hat sich die Welect GmbH ein solides Fundament im nationalen Heimatmarkt aufgebaut. So sehen bereits heute mehrere Millionen Menschen Werbung mit der Welect GmbH. Doch das Unternehmen will in den kommenden Jahren auch über den deutschen Markt hinauswachsen. Die Vision, welche die beiden Gründer über Produkte und Märkte hinweg umsetzen, soll jedoch unverändert bleiben.

\section{Werbebranche im Wandel}

Im Zeitraum von 2015 bis 2017 verzeichnete die Werbebranche in Deutschland eine positive Gesamtentwicklung, wobei sich die Bruttowerbeausgaben im Jahr 
2015 um 3,5 Prozent auf 29,2 Milliarden Euro erhöhten; im Jahr 2016 erfolgte ein erneuter Anstieg um 4,9 Prozent auf 30,9 Milliarden Euro. ${ }^{1}$ Auch in 2017 konnte eine Steigerung der Bruttowerbeausgaben gegenüber dem Vorjahr verzeichnet werden: Insgesamt gaben deutsche Unternehmen 31,8 Milliarden Euro für Werbung aus, was einer Steigerung von 1,8 Prozent entspricht.

Laut dem Werbemarktforschungsunternehmen Nielsen entfiel der höchste Anteil der Werbeausgaben in den Jahren von 2015 bis 2017 auf Fernsehwerbung (2015: 13,8 Milliarden Euro; 2016: 14,9 Milliarden Euro; 2017: 15,3 Milliarden Euro). Die Ausgaben für Fernsehwerbung stiegen dabei über die betrachtete Zeitperiode von Jahr zu Jahr an (2015: +5,4 Prozent; 2016: +6,8 Prozent; 2017: +1,6 Prozent), genau wie die Ausgaben für Out of home/Außenwerbung beispielsweise über Großbildschirme oder Plakate ( $+5,4$ Prozent; $+6,8$ Prozent; $+1,6$ Prozent) und die Ausgaben für Radiowerbung (+2,9 Prozent; +8,9 Prozent; +4,6 Prozent). Das stärkste Wachstum verzeichneten allerdings die Ausgaben für Werbung auf mobilen Endgeräten (+58,0 Prozent; +72,4 Prozent; +32,9 Prozent).

Weniger eindeutig verlief in den Jahren von 2015 bis 2017 die Entwicklung der Ausgaben für Werbung im Internet (+0,1 Prozent; -4,2 Prozent; -2,5 Prozent) und Werbung in Printmedien, das heißt Publikumszeitschriften, Fachzeitschriften und Zeitungen (-0,8 Prozent; $+1,5$ Prozent; $-1,1$ Prozent). Großen Schwankungen unterlagen auch die Ausgaben für Kinowerbung (+20,2 Prozent; -0,4 Prozent; $+7,8$ Prozent). ${ }^{2}$

Einen Überblick der Werbeausgaben im Zeitraum von 2015 bis 2017 in den jeweiligen Werbekanälen zeigt Tabelle 9.1.

Die Zahlen spiegeln eine Veränderung in der Struktur der Werbebranche wider. So verlor insbesondere die Werbung über Printmedien von 2015 bis 2017 an Bedeutung, während diejenige über mobile Endgeräte zunehmend wichtiger wurde. Die Statistik erfasste jedoch nicht moderne Werbekanäle wie Social-MediaMarketing oder Influencer-Marketing, deren Bedeutung in den Jahren von 2015 bis 2017 ebenfalls zunahm. ${ }^{3}$

Der Trend der zunehmenden Digitalisierung der Werbebranche zeigt sich jedoch nicht nur in der Bedeutung der klassischen Werbekanäle und der Entste-

1 Vgl. GENIOS (2016), verfügbar unter http://www.genios.de/document/BRAW_r_mar_ 20161116; Nielsen Insights (2016), verfügbar unter https://www.nielsen.com/de/de/pressroom/2016/Werbemarkt-2015-verzeichnete-ein-Plus.html; GENIOS (2017), verfügbar unter https://www.genios.de/document/BRAW_r_mar_20171116.

2 Vgl. Nielsen Insights (2018), verfügbar unter https://www.nielsen.com/de/de/insights/news/ 2018/wp-02-werbejahr2017.html.

3 Vgl. Absatzwirtschaft (2015), verfügbar unter http://www.absatzwirtschaft.de/digitalmarket ing-mehr-als-social-media-und-banner-werbung-60581/. 
Tab. 9.1: Bruttowerbeausgaben insgesamt und nach Kanälen (von 2015 bis 2017)

\begin{tabular}{|c|c|c|c|c|c|c|}
\hline & \multicolumn{2}{|c|}{2015} & \multicolumn{2}{|c|}{2016} & \multicolumn{2}{|l|}{2017} \\
\hline & $\begin{array}{l}\text { Ausgaben }{ }^{\mathrm{a}} \\
\text { (Milliarden) }\end{array}$ & $\begin{array}{l}\Delta^{\mathrm{b}} \\
(\%)\end{array}$ & $\begin{array}{r}\text { Ausgaben } \\
\text { (Milliarden) }\end{array}$ & $\begin{array}{r}\Delta \\
(\%)\end{array}$ & $\begin{array}{r}\text { Ausgaben } \\
\text { (Milliarden) }\end{array}$ & $\begin{array}{r}\Delta \\
(\%)\end{array}$ \\
\hline Insgesamt & 29,2 & $+3,5$ & 30,9 & $+4,9$ & 31,8 & $+1,8$ \\
\hline Fernsehen & 13,8 & $+5,4$ & 14,9 & $+6,8$ & 15,3 & $+1,6$ \\
\hline Zeitungen & 4,7 & $-0,2$ & 4,9 & $+4,0$ & 4,9 & $-0,5$ \\
\hline Publikumszeitschriften & 3,5 & $-1,8$ & 3,5 & $-1,8$ & 3,4 & $-1,5$ \\
\hline Internet & 3,0 & +0.1 & 2,9 & $-4,2$ & 2,9 & $-2,5$ \\
\hline Out-of-Home & 1,5 & $+9,7$ & 1,8 & $+7,9$ & 2,2 & $+12,4$ \\
\hline Radio & 1,7 & $+2,9$ & 1,8 & $+8,9$ & 1,9 & $+4,6$ \\
\hline Fachzeitschriften & 0,4 & $+0,5$ & 0,4 & $+1,7$ & 0,4 & $-4,8$ \\
\hline Mobile & 0,3 & $+58,0$ & 0,5 & $+72,4$ & 0,6 & $+32,9$ \\
\hline Kino & 0,1 & $+20,2$ & 0,1 & $-0,4$ & 0,2 & $+7,8$ \\
\hline
\end{tabular}

${ }^{a}$ Bruttowerbeausgaben (in Euro, Werte gerundet)

${ }^{b}$ Relative Veränderung zum Vorjahr (in Prozent)

Quelle: GENIOS (2016), verfügbar unter http://www.genios.de/document/BRAW__r_mar_ 20161116;

GENIOS (2017), verfügbar unter https://www.genios.de/document/BRAW_r_mar_20171116; Nielsen Insights (2016), verfügbar unter https://www.nielsen.com/de/de/press-room/2016/

Werbemarkt-2015-verzeichnete-ein-Plus.html;

Nielsen Insights (2018), verfügbar unter https://www.nielsen.com/de/de/insights/news/ 2018/wp-02-werbejahr2017.html.

hung Neuer, er äußert sich auch in Branchentrends. So liefern die Angaben zu den deutschen Bruttowerbeausgaben - welche Nielsen zur Verfügung stellt - außerdem Informationen zu den werbestärksten Branchen in den Jahren von 2015 bis 2017. An der Spitze des Branchen-Rankings lagen demnach E-Commerce und Online-Dienstleistungen, aber auch die Automobilbranche, der Lebensmitteleinzelhandel sowie die Arzneimittelbranche zählten zu den werbeintensiven Branchen.

Die Digitalisierung von Werbung und Marketing wurde in den Jahren von 2015 bis 2017 zudem von Veränderungen in der Anbieterlandschaft begleitet. Diese Veränderungen beschränkten sich nicht nur auf den deutschen Markt und werden den internationalen Werbemarkt - erwartungsgemäß - auch in den kommenden Jahren stark beeinflussen. So nimmt der Wettbewerbsdruck durch neue Akteure wie Unternehmensberatungen und große Internetkonzerne zu, die immer stärker in das Kerngeschäft von Werbung und Marketing vordringen. Fusionen und Über- 
nahmen zählen zum Tagesgeschäft der Branche. ${ }^{4}$ Insbesondere die Konkurrenz durch Konzerne wie Google, Facebook und Amazon verdeutlicht darüber hinaus das für die Werbebranche wichtige Thema der Erfassung und Nutzung von Daten im Werbekontext.

Um im Wettbewerb bestehen zu können, müssen Akteure nicht selten ihr eigenes Profil anpassen bzw. schärfen. Neben einer Media-Expertise sind zunehmend Kompetenzen wie technologisches Know-how, aber auch Flexibilität und Fantasie gefragt. Insbesondere im deutschen Markt gilt dies sowohl für die großen Mediaagenturen als auch für die zahlreichen kleinen Werbeunternehmen.

\section{Entstehung eines Adtech-Start-ups}

Am 21.März 2016 gründeten Philipp Dommers und Olaf Peters-Kim das AdtechUnternehmen Welect GmbH. Diesem Tag ging eine Phase voraus, in welcher sich die Gründer intensiv mit der Idee auseinandersetzten, „Werbung neu zu erfinden“. Bis zur tatsächlichen Gründung sollte etwa ein Jahr vergehen, in welchem sie ihre Idee entwickelten.

Nach seinem Abschluss zum Diplom-Ingenieur mit dem Schwerpunkt Media Technologies und ersten Erfahrungen in der Medienbranche begann Dommers im Jahr 2009 als Trainee bei einer Mediaagentur, wo er zunächst einer klassischen Beraterlaufbahn folgte. Bereits ab 2007 arbeitete auch Peters-Kim als CFO für dieselbe Agentur. Als Teil ihrer Tätigkeit dort beschäftigten sich beide damit, wie Werbung in den unterschiedlichen Kanälen funktioniert und wie Menschen Werbung wahrnehmen. Dabei fiel ihnen auf, dass „insbesondere in der digitalen Welt bislang sehr wenig Rücksicht darauf genommen wird, ob ein Nutzer überhaupt offen ist für werbliche Kommunikation oder nicht“. Dommers vergleicht die Voraussetzungen für wirksame digitale Werbungen mit denen eines normalen Alltagsgespräches: „Kommunikation kann nur funktionieren, wenn beide Seiten auch Lust dazu haben."

Anfang 2015 waren sich Dommers und Peters-Kim einig über die Notwendigkeit eines Systems, welches es Menschen erlaubt, selbst $\mathrm{zu}$ entscheiden, ob sie Werbung sehen möchten oder nicht. Es folgten intensive Monate, in denen sie an ihrer Idee arbeiteten, anfangs in ihrer Freizeit. Schnell wurde beiden klar, dass sie sich selbstständig machen wollten. So beendeten sie ihre Tätigkeit in der Agentur und gründeten das Unternehmen Welect $\mathrm{GmbH}$.

4 Vgl. Horizont (2017), verfügbar unter https://www.horizont.net/agenturen/kommentare/Wastun-Kapituliert-die-Agenturbranche-vor-der-uebermacht-der-Berater-161567. 


\section{Voneinander lernen}

Mit Dommers und Peters-Kim entschieden sich zwei Mediaexperten dafür, gemeinsam ein Unternehmen zu gründen. Beide profitierten insbesondere in der Anfangsphase von ihren Erfahrungen in der Werbebranche, aber ebenso wichtig waren ihre unterschiedlichen Kompetenzen, die sie einbrachten: Peters-Kim aus dem Bereich Finance, Dommers aus der Kundenberatung und dem Management von Kampagnen.

Doch die Gründer lernten nicht nur voneinander, sondern waren auch aufgeschlossen gegenüber externer Hilfe: „Wir haben uns bei sehr vielen Menschen Hilfe geholt und auch immer wieder nach Hilfe gefragt. Als Erstes haben wir uns, was unsere Idee betrifft, so früh wie möglich [...] mit Experten ausgetauscht. Das heißt, wir haben sehr viele Interviews mit alten Kollegen, mit Experten, mit Forschungsabteilungen geführt, um herauszufinden, ob das Vorhaben überhaupt grundsätzlich funktionieren kann.“

Externes Wissen nahmen sie insbesondere für das Thema Entwicklung in Anspruch. Dies erfolgte - trotz des Verständnisses der Gründer für Technologie - in Form einer Kooperation mit dem Softwareentwickler 9elements in Bochum, der sich unter anderem auf das Projektgeschäft mit Start-ups konzentriert.

\section{Standort Düsseldorf}

Ausschlaggebend für die Wahl des Standorts Düsseldorf war - neben der persönlichen Verbundenheit mit der Stadt - die Nähe zum Kunden/zur Kundin. Große Werbeagenturen wie die GroupM Germany oder OMD haben hier ihren Sitz und verwalten von hier aus ihre Werbebudgets. Ebenso wichtig war: „Die Start-up-Szene in Düsseldorf ist in den letzten Jahren stark gewachsen [...] man kann sich in dem Bereich sehr gut austauschen.“

\section{Überregionales Wachstum von 2016 bis 2018}

Laut der Gründer der Welect $\mathrm{GmbH}$ ist das mediale Nutzungsverhalten heute stark selbstbestimmt und die Mehrzahl der Verbraucher entscheidet sich aktiv dafür, welche Medien und Inhalte sie wann, wie und wo konsumieren. „Die meisten Menschen schauen mittlerweile abends auf der Couch eher Netflix, Amazon Prime oder YouTube und die wenigsten gucken den Blockbuster auf Pro7.“ Der Ansatz der Welect $\mathrm{GmbH}$ besteht darin, Menschen selbst entscheiden $\mathrm{zu}$ lassen, ob sie Werbung sehen möchten und welche sie interessiert. 
Das Unternehmen führte sogleich im Jahr 2016 regional die App WelectGo ein, die es so inzwischen nicht mehr gibt. Stattdessen liegt der Fokus seit 2017 auf dem Dienst WelectPublish, über welchen bereits heute mehrere Millionen Menschen pro Monat Werbung sehen. Mittel- bis langfristig gehen nach Einschätzung der Gründer mit diesem Kernprodukt weitere nationale als auch internationale Wachstumschancen einher.

\section{WelectGo}

Die App WelectGo bot Nutzern des öffentlichen Personennahverkehrs die Option, auf werbefinanzierte Tickets zuzugreifen. Sie erhielten ihre Fahrkarte kostenlos, sofern sie bereit waren, sich im Gegenzug ein Werbevideo anzuschauen. Zum Einsatz kam WelectGo hierbei bei der Düsseldorfer Rheinbahn und anderen Verkehrsbetrieben. Der Ansturm auf die verfügbaren Fahrkarten überstieg die Erwartungen der Gründer: „Die App ist tatsächlich über Nacht bekannt geworden, [...] wir haben das so nicht geplant.“ Innerhalb kürzester Zeit meldeten sich Zehntausende an. Im Dezember 2016 schaffte es die App sogar unter die Top 10 der AppStore-Charts. Die Welect GmbH erhielt große Aufmerksamkeit und war in den lokalen Medien stark vertreten, unter anderem berichteten die Rheinische Post, Antenne Düsseldorf, WDR und RTL West über das Adtech-Start-up. Die App setzte das Ziel des Unternehmens um: Nutzer erhielten die Möglichkeit, sich selbstbestimmt für den Konsum von Werbung zu entscheiden - und verknüpften diese mit einem positiven Erlebnis.

WelectGo stellte die Gründer zugleich vor neue Herausforderungen, denn die App war nicht ohne Weiteres skalierbar. Die individuelle Organisation der jeweiligen Verkehrsbetriebe führte zu der Schwierigkeit, WelectGo technisch einheitlich umzusetzen. Darüber hinaus stellten die Ticketpreise - von 1,60 bis 2,70 Euro - aus Sicht der Werbebranche relativ hohe Beträge dar, deren Finanzierung das Start-up über werbetreibende Unternehmen zunächst sichern musste. Obwohl infrastrukturell nicht darauf vorbereitet, gelang es dem Team, über ein Jahr lang auf Projektbasis neue Kampagnen für den lokalen Raum zu akquirieren, um die hohe Nachfrage zu befriedigen. Dann trafen die Gründer die Entscheidung, das Projekt zu beenden: „Wir haben natürlich auch sondiert, ob man die App langfristig über mehrere Jahre spielen kann. Insbesondere, als die App so erfolgreich war, haben wir diese Möglichkeit selbstverständlich diskutiert. Letzten Endes sind wir dann doch bei dem Plan geblieben, das Projekt nach einem bestimmten Zeitraum zu beenden.“

Insgesamt wurden über WelectGo 70.000 Tickets vergeben und 50.000 Nutzer erreicht. Die Technologie dieser App kommt nach wie vor zum Einsatz, indem eine 
Lizenz vergeben wurde. Die Installation in eine bestehende App erlaubt es der Welect GmbH, die Technik zwar anzubieten, aber den Vertrieb auf lokaler Ebene anderen zu überlassen.

\section{WelectPublish}

Seit 2017 liegt der Fokus der Welect GmbH auf einem anderen Produkt: den Online-Dienst WelectPublish. Dommers und Peters-Kim hatten es bereits vor dem Aus der App entwickelt und sahen in diesem Dienst nun ihr Kernprodukt.

Von zentraler Bedeutung für WelectPublish ist die Zusammenarbeit mit Verlagen, welche ihre redaktionellen Inhalte auf Online-Portalen zur Verfügung stellen. Eine spezielle Technologie, die auf einer unabhängigen und selbst entwickelten Plattform basiert, ermöglicht die Honorierung redaktioneller Inhalte. Nutzer erklären sich dazu bereit, ein Werbevideo ihrer Wahl anzuschauen. Damit können sie die redaktionelle Arbeit freiwillig honorieren oder ihnen steht die Option zur Verfügung, dadurch übliche Barrieren wie Paywall, AdBlock-, Anmelde- und Aboschranken zu überwinden.

WelectPublish verfolgt das Ziel, ,dass alle drei Zielgruppen profitieren“. Die erste Zielgruppe, die Verbraucher, entscheidet, welche Werbung sie sehen möchte und welche nicht. Das ist eine Option, die insbesondere bei Add-Block-Nutzern sehr gut funktioniert.

Die zweite Zielgruppe, die Verlage, erhält durch WelectPublish eine neue Erlösquelle. Insbesondere Leser/innen, die sich von Paywall, AdBlock-, Anmeldeund Aboschranken abschrecken lassen, können so für redaktionelle Inhalte erreicht werden. Bislang hat die Welect $\mathrm{GmbH}$ unter anderem Partner wie Rheinische Post Online, FOCUS Online und CHIP gewonnen.

Als dritte Zielgruppe profitieren die Werbetreibenden von WelectPublish. Da Nutzer entscheiden, sich ein Werbevideo anzusehen, könnten sie offen für werbliche Kommunikation sein. Aufgrund der Option, einen Werbespot abzubrechen, entstehen Kosten für den Werbetreibenden tatsächlich nur dann, wenn ein Video vollständig geschaut wird. „Organisches Targeting“ stellt außerdem eine Affinität zum jeweiligen Werbespot sicher. Der Nutzer kann also den Spot auswählen, der für ihn in diesem Moment am relevantesten ist.

\section{Wachstum in alle Richtungen}

Mit dem Kernprodukt WelectPublish möchte das Unternehmen weiterhin wachsen. Damit verbunden ist auch eine Vergrößerung des Teams. Im August 2018 
bestand es aus vier Mitarbeiter/innen. Weitere Einstellungen sind in Zukunft geplant. Dabei soll das Wachstum nicht exponentiell, sondern eher konservativ erfolgen. Besonderen Wert legen die Gründer auf die sorgfältige Auswahl besonders qualifizierter Mitarbeiter/innen mit der notwendigen Motivation und Teamfähigkeit.

Dommers und Peters-Kim erwarten aufgrund der Skalierbarkeit von WelectPublish ein langfristiges Wachstum ihres Unternehmens in internationalen Märkten. „Der nächste Markt ist für uns sicherlich interessant. Das ist zum einen UK aber auch die Nordic-Länder. Und tatsächlich ist das auch etwas, das wir in den nächsten 24 Monaten sehr konkretisieren werden." Ausschlaggebend für die Auswahl der Zielmärkte sind in erster Linie gute persönliche Kontakte zu Geschäftspartnern vor Ort, beispielsweise in London. Für die Auswahl der Zielmärkte spielt zudem eine Vergleichbarkeit zum deutschen Markt eine Rolle: „Der UK-Markt ist dem deutschen Markt sehr ähnlich [...]. Das heißt, wenn wir in Deutschland die Mechaniken, die wir gerade etablieren, ins Rollen gebracht haben, dann ist es sehr leicht, diese auch in UK zu installieren." Die Art des internationalen Markteintritts wird stark von den Eigenschaften des Produkts WelectPublish bestimmt sein. Unabhängig davon, ob es sich um Aktivitäten im In- oder Ausland handelt, geht es im ersten Schritt immer darum, Verlage als Partner zu gewinnen. Erst im zweiten Schritt erhalten werbetreibende Akteure ein Angebot für entsprechende Werbeflächen. Auf diese Art und Weise erfolgte die Skalierung innerhalb Deutschlands, genauso kann ein Wachstum in anderen europäischen Ländern wie Großbritannien - erreicht werden. Insgesamt betrachtet führt die relativ einfache Skalierbarkeit des Produkts, die Ähnlichkeit potenzieller Zielmärkte sowie das Vorhandensein von ausländischen Geschäftskontakten dazu, Risiken einer internationalen Expansion zu reduzieren.

Entscheidend für das Wachstumspotenzial und die langfristige Wettbewerbsfähigkeit der Welect $\mathrm{GmbH}$ - national wie international - ist die Erkenntnis, welche Vorteile es hat, den Verbraucher/die Verbraucherin in die Werbung einzubeziehen. Diese Erkenntnis ist insofern innovativ, weil das Thema der nutzerzentrierten Werbung zum Zeitpunkt der Gründung der Welect $\mathrm{GmbH}$ noch keine große Rolle gespielt hat. Auch nach wie vor liegt der Fokus vieler Großkonzerne im Bereich Programmatic Advertising, dem automatischen und individualisierten Ein- und Verkauf von Werbeflächen auf der Grundlage vorliegender Nutzerdaten. Verschiedene Algorithmen sollen ermitteln, was den Verbraucher/die Verbraucherin vermutlich interessiert.

Wie stark die konventionelle Werbung nach wie vor in den Köpfen von Verbrauchern/innen und Werbetreibenden vorherrscht, verdeutlicht Dommers mit einer Anekdote. Im Rahmen einer Präsentation bat er seine Zuhörer/innen um Ideen, wie man in der digitalen Welt die Lieblingsfarbe einer Person herausfin- 
den kann. Unter den Vorschlägen waren unter anderem die Analyse des letzten Warenkorbs oder der Einsatz von Cookies. Niemand kam jedoch auf die Idee, Personen in der digitalen Welt direkt nach der Lieblingsfarbe $\mathrm{zu}$ fragen. Hier setzt das Prinzip der nutzerzentrierten Werbung durch die Welect $\mathrm{GmbH}$ an.

Dieser Ansatz steht dem Vorgehen vieler Kampagnen gegenüber, die vorwiegend „data-driven“ und weniger „data-informed“ entscheiden, ob ein Werbespot für einen bestimmten Nutzer/eine Nutzerin relevant ist oder nicht. Dabei besteht bei Kampagnen, die data-driven umgesetzt werden, die Gefahr, wichtige Aspekte bei den Bedürfnissen von Menschen außer Acht zu lassen. Bei der Welect $\mathrm{GmbH}$ wird die nutzerzentrierte Werbung konsequent umgesetzt und die individuellen Entscheidungen der Menschen, die auch Erfahrungen, Intuition und Gefühle beinhalten, stehen im Vordergrund.

Für die weitere Entwicklung des Unternehmens ist es vorteilhaft, dass die Welect $\mathrm{GmbH}$ bereits heute ohne die Erfassung sensibler Nutzerdaten auskommt. Die Vorteile einer nutzerzentrierten Werbung werden angesichts der aktuellen E-Privacy-Debatte besonders evident, denn das Sammeln großer Datensätze entfällt, um den Nutzer/die Nutzerin mit dem passenden Content zu versorgen. Dies ist ein wesentlicher Wettbewerbsvorteil, falls es $\mathrm{zu}$ datenschutzrechtlichen Gesetzesänderungen kommen sollte.

\section{Kurz und bündig}

Die Bedeutung der nutzerzentrierten Werbung veranlasste Dommers und PetersKim im Jahr 2016 dazu, das Düsseldorfer Adtech-Start-up Welect GmbH zu gründen. Dem Ziel, Werbung auf die Verbraucher/innen auszurichten und zu diesem Zweck eine direkte Kommunikation mit diesen anzustreben, folgte zunächst die Entwicklung der App WelectGo. Aber auch der Online-Service WelectPublish, der seit 2017 das Kerngeschäft des Start-ups darstellt, verfolgt dieses Ziel.

Mit der Fokussierung auf WelectPublish konnte sich das Unternehmen in Deutschland etablieren. Im Hinblick auf die Zukunft sehen die Gründer ein weiteres Wachstumspotenzial auch in ausgewählten internationalen Märkten. Sie wissen, dass sie nicht alles perfekt planen können: „Wir sind kein Unternehmen, was mit einem Fünf-Jahres-Plan arbeitet. Dafür verändert sich der Markt zu schnell, und dafür sind wir auch zu spontan." Stattdessen vertrauen sie lieber auf Flexibilität und Anpassungsvermögen - zwei Fähigkeiten, die sie für ihr Unternehmen bereits in der Vergangenheit unter Beweis stellen konnten. 


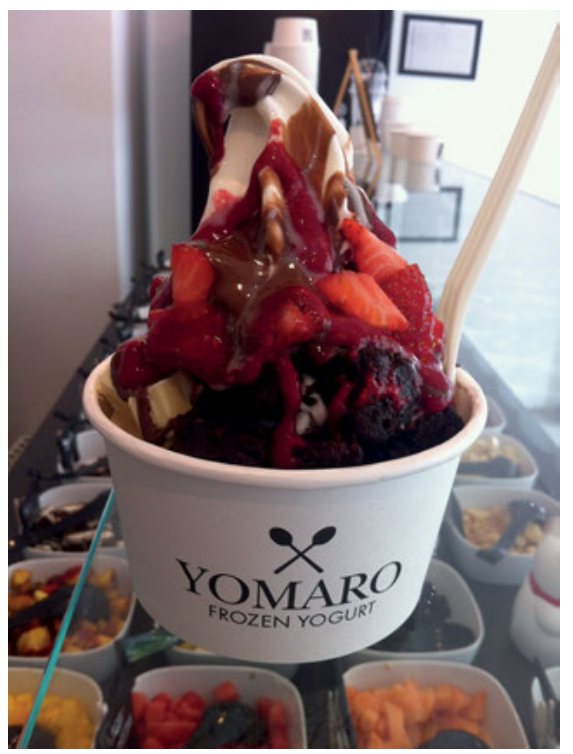

Frozen Yogurt von YOMARO

(Bildnachweis: YOMARO GmbH) 